\title{
Structure Stabilizing Role of Aromatic Interactions is Decided by Spatial Arrangement of Aromatic Pairs: A Case Study With Designed Peptide $\beta$-Hairpins
}

\author{
Kamlesh Madhusudan Makwana and Radhakrishnan Mahalakshmi* \\ Molecular Biophysics Laboratory, Department of Biological Sciences, Indian Institute of \\ Science Education and Research, Bhopal, 462023, India
}

\section{Introduction}

Interaction among aromatic side chains in the hydrophobic core of proteins is a well-known mechanism for protein structure stabilization [1]. Aromatic pairs at the non-hydrogen bonding position of designed $\beta$-hairpin peptides interact by $\mathrm{T}$-shaped arrangement and usually result in structured folds [2]. However, the consequences of spatial positioning and preferred packing geometry of aromatic pairs on scaffold structure and stability are still unclear. Studies on tryptophan packing geometry have revealed that the heterogeneous indole ring preferentially occupies "face" geometry in T-shaped aromatic interactions [3]. But the fate of Trp at the sterically constrained "edge" geometry is not well understood. Using $\beta$-hairpin peptide models we describe here a detailed investigation highlighting the implication of aromatic-aromatic interactions in the formation and measured stability of $\beta$-hairpin scaffolds.

\section{Results and Discussion}

Octapeptide $\beta$-hairpins nucleated by ${ }^{\mathrm{D}} \mathrm{P}-\mathrm{G}$ segment and with a single non-hydrogen bonding position [4], were chosen as model systems to probe all possible aryl pair interactions involving Phe, Tyr, and Trp. All peptides were synthesized using Fmoc-chemistry on a Rink amide resin (Figure 1a). Being hydrophobic in nature, the peptides were highly soluble in methanol but not in water. This feature allowed us to investigate the preferred modes of aromatic interactions in the absence of solvent-driven hydrophobic contributions, using high-resolution NMR and CD spectroscopy. Complete assignment of the resonances was achieved using proton 1D, 2D ROESY, TOCSY and heteronuclear HSQCTOCSY experiments. The spectra revealed that all the peptides adopted $\beta$-hairpin structures and gave rise to NOEs characteristic of $\beta$-hairpins. Comparison of fraction folded populations (Figure 1b), extent of signature downfield shifted backbone resonances, extent of glycine splitting and HPLC retention time, and anomalous chemical shifts for resonances influenced by aromatic ring current effects (Figure 2a) revealed the stabilizing and destabilizing contribution of tryptophan.

Positional permutants YW and FW pairs displayed an interconversion of Face-to-Edge $(\mathrm{FtE})$ and Edge-to-Face (EtF) geometry, resulting in scaffold destabilization (Figure 2b). However, we found evidence of a single FtE geometry in the analogs WY and WF pairs, with the highest stabilizing contribution of aromatic interactions to the $\beta$-hairpin scaffold.

(a)

\begin{tabular}{|c|c|}
\hline Peptide & Sequence \\
\hline YY & AC-L-Y-V- ${ }^{D}$ P-G-L-Y-V-NH \\
\hline FY & Ac-L-F-V- ${ }^{D}$ P-G-L-Y-V-NH \\
\hline WY & Ac-L-W-V- ${ }^{\mathrm{D}} \mathrm{P}-\mathrm{G}-\mathrm{L}-\mathrm{Y}-\mathrm{V}-\mathrm{NH}_{2}$ \\
\hline $\mathrm{FF}$ & Ac-L-F-V- ${ }^{D}$ P-G-L-F-V-NH ${ }_{2}$ \\
\hline YF & AC-L-Y-V- ${ }^{D}$ P-G-L-F-V-NH \\
\hline WF & Ac-L-W-V- ${ }^{\mathrm{D}} \mathrm{P}-\mathrm{G}-\mathrm{L}-\mathrm{F}-\mathrm{V}-\mathrm{NH}_{2}$ \\
\hline YW & Ac-L-Y-V- ${ }^{D}$ P-G-L-W-V-NH ${ }_{2}$ \\
\hline FW & Ac-L-F-V- ${ }^{\mathrm{D}} \mathrm{P}-\mathrm{G}-\mathrm{L}-\mathrm{W}-\mathrm{V}-\mathrm{NH}_{2}$ \\
\hline WW & AC-L-W-V- ${ }^{D}$ P-G-L-W-V-NH \\
\hline
\end{tabular}

(b)

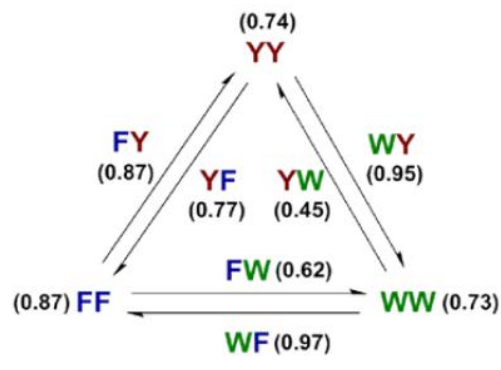

Fig. 1. Sequences of peptide $\beta$-hairpins. (b) Comparison of fraction folded populations across the peptides (provided in brackets). Reprinted (adapted) with permission from [5]. 
Our meticulous examination of all possible aryl-aryl interactions at a single non-hydrogen bonding position in $\beta$-hairpin scaffolds revealed a near-universal T-shaped Face-to-Edge mode of interaction among aromatic pairs. However, the indole ring, particularly in amphipathic solvents, causes destabilization of the $\beta$-hairpin structure when placed at the sterically constrained edge position. Thus, the implication of tryptophan, when interacting with non-Trp rings in $\beta$-hairpin stabilization can be contextual: stabilizing at "face" and destabilizing at "edge" geometry. Such small variations in the geometry may have a strong bearing in the hydrophobic protein interior. Our results serve as important criteria in the rational design of stable structural scaffolds.

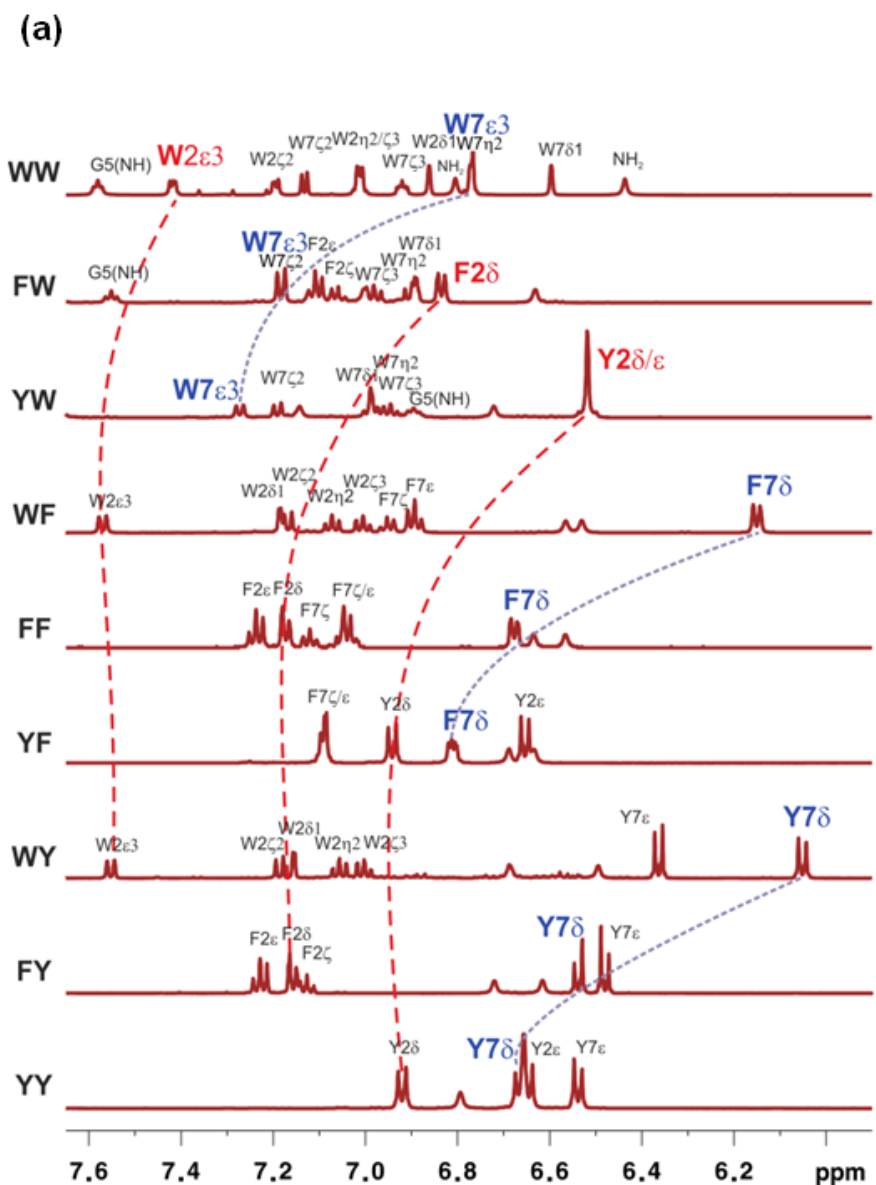

(b)

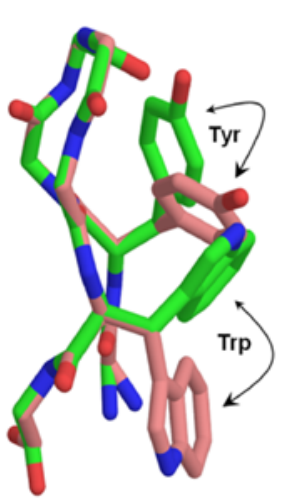

Fig. 2. (a) Comparison of signature upfield shifted aromatic proton resonances across all peptides. Stack plot of proton $1 D$ NMR spectra at $303 \mathrm{~K}$ in methanol. The upfield shifted protons, $F 7 \delta$ and $W 7 \varepsilon 3$ is a signature of FtE geometry. The significant upfield shifted protons $Y 2 \delta / \varepsilon, F 2 \delta$ and $W 2 \varepsilon 3$ in aro-Trp pairs is evidence for the flipped EtF geometry. Reprinted (adapted) with permission from [5]. (b) Modeled structure showing the interconversion of FtE to EtF, as what seen in Aro-W pairs. Reprinted (adapted) with permission from Makwana \& Mahalakshmi [6]. Copyright (2015) American Chemical Society. 


\section{Acknowledgments}

K.M.M. acknowledges the University Grants Commission, Govt. of India, for research fellowship. R.M. is a recipient of the Welcome Trust/DBT India Alliance Intermediate Fellowship. This work was supported by intramural funds. K.M.M thanks the Department of Science and Technology, Govt. of India for International Travel Support. K.M.M. also thanks the 24th American Peptide Symposium for the APS Travel Award.

\section{References}

1. Burley, S.K., Petsko, G.A. Science 229, 23-28 (1985), http://dx.doi.org/10.1126/science.3892686

2. Santiveri, C.M., Jimenez, M.A. Biopolymers 94,779-790 (2010), http://dx.doi.org/10.1002/bip.21436

3. Samanta, U., Pal, D., Chakrabarti, P. Acta Crystallogr. Sect. D Biol. Crystallogr. 55, 1421-1427 (1999), http://dx.doi.org/10.1107/S090744499900726x

4. Mahalakshmi, R., Raghothama, S., Balaram, P. J. Am. Chem. Soc 128, 1125-1138 (2006), http://dx.doi.org/10.1021/ja054040k

5. Makwana, K.M., Mahalakshmi, R. ChemBioChem 15, 2357-2360 (2014), http://dx.doi.org/10.1002/cbic.201402340

6. Makwana, K.M., Mahalakshmi, R. J. Phys. Chem. B 119, 5376-5385 (2015), http://dx.doi.org/10.1021/acs.jpcb.5b00554 